\section{A surprising muddle in the optic nerve}

\section{from Julian Lewis}

STUDENTS of the brain bewail its complexity. But how complex is it? Neurones can be classified into discrete categories according to their shape, location and connections; and though the number of such types is large - perhaps thousands - and the catalogue not yet complete, still the classification brings about a vast simplification. Neurones in the same category behave in similar ways, and make similar connections. But that is not to say that they make identical connections. The cells of a given category are spread over a range of neighbouring positions, and though they may all send their axons to the same target set of neurones, they do not send them all to precisely the same site within that target area, nor do they send them to sites distributed within it at random. It is usual rather to find an orderly map of one class of neurones onto another. The crucial feature of such a map is that it is continuous: neighbouring points of the one set project to neighbouring points of the other.

Since continous neural maps are so commonplace in the brain, and seemingly so fundamental to its plan, one might hope to discover some simple and general mechanism of cell behaviour by which they are all set up. This hope has not been realised. Twenty years ago it appeared perhaps that Sperry might have solved the problem with his theory of neuronal specificity, whereby each cell was deemed to be chemically distinct, and to connect selectively by matching its own chemical label with that of a correspondingly distinctive target cell. Evidence came mainly from experiments on fish and amphibians. In the most famous of these, the optic nerve of a newt was cut and its eye turned upside down. The nerve regenerated, visual connections re-formed, and the animal saw the world again; but it saw the world upside down. Sperry's arguments were ingenious and persuasive. But subsequent studies, especially of connections between retina and tectum, have undermined his theory, at least in its extreme form. It seems almost certain that the arrangement of retino-tectal connections depends on interactions between one presynaptic fibre and another, and not simply on a matching of a presynaptic with a postsynaptic label. Such interactions might indeed be expected $a$ priori to underlie the preservation of neighbourhood relationships which is the essence of a continous map. But if Sperry's theory does not seem entirely true, neither does it seem entirely false. Continuity alone

Julian Lewis is a Lecturer in the Department of Anatomy, King's College, London. is not enough to define, for example, the orientation of a map or the precise positions of its boundaries and landmarks, and chemical markers such as Sperry envisaged very probably play a part here.

As evidence has accumulated against the strict form of Sperry's theory, some absolutists have sought to reject it utterly, and enthrone in its place another allexplanatory principle. In particular, there has been a revival of the suggestion, discounted by Sperry, that orderly maps can be understood as a consequence of the rules of mechanical guidance of axon outgrowth (Holder \& Martin Symp. Soc. exp. Biol. XXXII, 275; 1978). That is, the axons may arrive at their targets in an orderly array because they have made the entire journey in an orderly array.

This idea has seemed plausible for connections between eye and brain, since there is evidence in many species that the axons all along the optic nerve are indeed ordered retinotopically. One of the most extraordinary instances has been described recently by Scholes (Nature 278, 620; 1979): in a cichlid fish, the optic nerve has the stucture of a ribbon pleated longitudinally, with the radial coordinate of position in the retina mapping across the breadth of the ribbon, and the circumferential coordinate mapping across its thickness. In monkeys and some other mammals, it has long been supposed from degeneration studies that there is again an ordering, though of a simpler sort (Polyak The Vertebrate Visual System, Chicago, 1957). In this issue of Nature (page 720), Horton, Greenwood and Hubel deal a blow to this belief, so far as adult cats are concerned at least. They inject a small spot of horseradish peroxidase into the lateral geniculate nucleus; the peroxidase is taken up by the optic axons terminating at the site of injection and is carried back to the retina, where it labeis the cells from which those axons derive. The labelled cell bodies form a small tight cluster. Thus fibres projecting to closely neighbouring points in the lateral geniculate originate from closely neighbouring cells in the retina. That much is no surprise. But in a handful of neurones the peroxidase labelling is so intense that the axons themselves can be followed in their courses; and it turns out that these axons, far from remaining neighbours throughout the optic nerve, diverge as they leave the retina and become dispersed among axons from other sites, only to converge to become neighbours again in the lateral geniculate. Electrophysiological tests corroborate this finding.

If there is order in the cat's optic nerve, it must be order of a very bizarre sort at best; though the example of the cichlid fish might make us hesitate to conclude that there is no order at all, but only a random interweaving of axons. It is nervertheless hard now to believe that the continous map from the cat's retina to its lateral geniculate could be the outcome of neighbourhood relationships maintained throughout the optic nerve.

The findings of Horton, Greenwood and Hubel may be construed by some as support for Sperry's ideas. But neuronal specificity is not the only device by which an orderly somatotopic map may develop despite disorder of the fibres en route from source to target. There are ways to establish continuity in a projection without having the neurones chemically individuated or their axons guided in parallel paths of outgrowth. In particular, electrical activity might play a part. Neighbouring cells in general will tend to be correlated in their time of firing. Thus a continous projection can be set up if axons which fire at the same time tend to make synapses on the same or on closely adjacent targets (Willshaw \& von der Malsburg Proc. Roy. Soc. B 194, 431; 1976). There is evidence for such a principle in the development of binocular neural connections in mammals and in amphibians (Keating Phil. Trans. Roy. Soc. $B$ 278, 277; 1977); and a similar idea has been invoked to explain associative learning.

But the history of the problem of neural maps teaches caution. The theories are many and seductive, and it is dangerous to yield entirely to the charms of one alone. Each may represent only a part of the truth. Different vertebrate species, and different neural subsystems, may make their maps differently.

\section{Reinnervation of adult muscle}

\section{from Clarke $R$. Slater}

THE neural control of muscle activity in vertebrates is based on a simple pattern of cellular connections. The highly branched axon of each motor nerve cell provides the exclusive innervation for each of a large number of muscle fibres. The simplicity and apparent stability of this pattern conceals considerable potential for adaptive change which is revealed when death or injury of motor neurones leaves some muscle fibres denervated. For then sprouts grow from the specialised terminals of the intact motor axons and reinnervate many of the denervated muscle fibres, often precisely at the site of the original nerve-muscle junction. This ability to compensate in the periphery for damage to motor neurones in the spinal cord is one of great practical significance not only in disease but also during ageing, for most of us will lose more than half our motor-neurones by the time we die. Although the nature of the signals which 